\title{
Magnetic Flux Diffusion Through HTS Shields
}

\author{
A. D. Havenhill, K. W. Wong, and C. X. Fan
}

\begin{abstract}
Slow field leakage in a polycrystalline superconducting cupshield placed in an external axial field $\left(H_{\text {ext }}\right)$ much weaker than $H_{C 1}$ shows a diffusive time dependence with a time scale of $10^{2} \mathrm{~s}$. As the field strength increases but is still less than $H_{C 1}$, a logarithmic time dependence of $10^{2} \mathrm{~s}$ time scale is observed. It is shown that this logarithmic time dependence is a result of flux line diffusion between superconducting grains rather than fluxoid avalanches or flux bundle creep.
\end{abstract}

\section{INTRODUCTION}

A polycrystalline superconducting magnetic cupshield (hollow cylinder with one end closed) was placed in an external axial field $\left(H_{\text {ext }}\right)$, and the field inside the shield $\left(H_{\text {int }}\right)$ was measured as a function of time. $H_{\text {ext }}$ was chosen near the critical threshold of the shield. Empirical curve fitting is consistent with a largely diffusive mechanism of flux leakage. The mechanism we propose involves flux lines that exist between the grains. The field is low and in the regime of the Meissner effect. Flux motion is statistical but tends to be in the direction of decreasing flux density, much like the diffusive motion of chemical species tends to be in the direction of decreasing concentration. As the motion is statistical and the flux density gradient is limited by the low field regime, flux diffusion is expected to be a slow process. Data collected using a shielding device in an applied field much larger than the critical threshold suggest a logarithmic time dependence. Since both shields show field leakage on similar time scales $\left(\sim 10^{2} \mathrm{~s}\right)$ we suspect that the mechanism may be similar. In this paper we show that a logarithmic time dependence at a high applied field is consistent with flux diffusion. The slow field leakage associated with polycrystalline superconducting material could have a significant effect on the operation of devices made from such material.

The mixed state of a Type II superconductor contains magnetic flux lines in the form of quantized fluxoids, each having flux $\Phi_{o}[1]$. The motion of fluxoid bundles (flux creep) in the presence of a magnetic pressure gradient is described well by the Kim-Anderson model [2]. Thus the mechanism of flux creep involving bundles of fluxoids moving from pinning site to pinning site occurs in $H>H_{C 1}$ and $T<T_{0}$, where $H_{C 1}$ is the lower critical field and $T_{0}$ is the lattice-glass transition temperature $\left(T_{0}\right.$ is generally $\left.\ll T_{C}\right)$. The activation

Manuscript received February 26, 1997; revised January 9, 1998.

A. D. Havenhill was with the Department of Physics and Astronomy, University of Kansas, Lawrence, KS 66045 USA. He is currently with the Department of Earth Sciences and Physics, University of South Dakota, Vermillion, SD 57069 USA.

K. W. Wong is with the Department of Physics and Astronomy, University of Kansas, Lawrence, KS 66045 USA.

C. X. Fan is with Midwest Superconductivity, Inc., Lawrence, KS 66049 USA.

Publisher Item Identifier S 1051-8223(98)04227-4. energy required for a fluxoid bundle to leave a pinning site is supplied by thermal energy [2]. Motion to another pinning site is aided by the Lorentz force, $\mathbf{J ~ X ~ B ~ [ 2 ] . ~ I f ~} \mathbf{J}$ and $\mathbf{B}$ are low enough, the rate of flux creep approaches zero ("critical state") [2]. Theoretical work done by Anderson [2] predicts that supercurrents will decay logarithmically. This implies that the internal field has a logarithmic time dependence which was found experimentally by Kim et al. [3]. The bundle hopping rate in the Kim-Anderson model is taken to be $\sim 10^{5}-10^{11} \mathrm{~s}^{-1}$ [4] so the time scale is $\sim 10^{-11}-10^{-5} \mathrm{~s}$. Fast movements of bundles of 50 or more fluxoids (avalanches) have been detected and monitored in the vortex state in the presence of a slowly ramped applied field by Field $e t$ al. [5]. Fluxoid avalanches have often been compared to avalanches of metastable sandpiles [5]-[7]. Shi and Wang [8] have shown that a self-organized criticality model for flux avalanche dynamics predicts a logarithmic time dependence. For our data that were taken at high applied field (70-90 G), it is therefore reasonable to expect avalanche behavior to occur, thus consistent with the logarithmic time dependence.

Although we found a logarithmic time dependence when the field is high (but still lower than $H_{C 1}$ ), our time scale is several orders of magnitude larger than that of the Kim-Anderson or avalanche models. In this paper we show that near the critical threshold of a polycrystalline shield which is much lower than $H_{C 1}$, the internal field supports a diffusive model, and that at higher applied fields a logarithmic time dependence (which we have seen) is still compatible with a flux diffusion model. In our model the critical state equation [9], [10] is used to derive a time dependent critical current density.

\section{EXPERIMENTAL SETUP}

The superconducting cupshield was made at Midwest Superconductivity, Inc. by coating a hollow $\mathrm{MgO}$ cylinder (closed at one end) with $\mathrm{Tl}_{2} \mathrm{Ba}_{2} \mathrm{Ca}_{2} \mathrm{Cu}_{3} \mathrm{O}_{10}$ (Tl-2223) thick film on both the inner and outer surface as shown in Fig. 1. To coat the $\mathrm{MgO}$ cylinder, $\mathrm{Tl}-2223$ was ground to powder of average grain size of $\sim 1-5 \mu \mathrm{m}$ using an agate mortar and pestle. The powder was then mixed with an organic binder, brushed on the surface of the $\mathrm{MgO}$ cylinder, and left to stand for several hours to flow and become near uniform in thickness. The cylinder was then heated in $\mathrm{O}_{2}$ at $\approx 400{ }^{\circ} \mathrm{C}$ for several hours so as to oxidize and volatilize the organic material leaving a coating of Tl-2223. The sample was subsequently heated at $850-900{ }^{\circ} \mathrm{C}$ in $\mathrm{O}_{2}$ for a short time to improve the film density and grain-grain contacts.

The dimensions of the $\mathrm{MgO}$ cylinder are given as follows:

$$
\begin{aligned}
& \text { Length }=12.7 \mathrm{~cm} \\
& \text { Outside diameter }=3.18 \mathrm{~cm} \\
& \text { Thickness }=5-6 \mathrm{~mm} .
\end{aligned}
$$




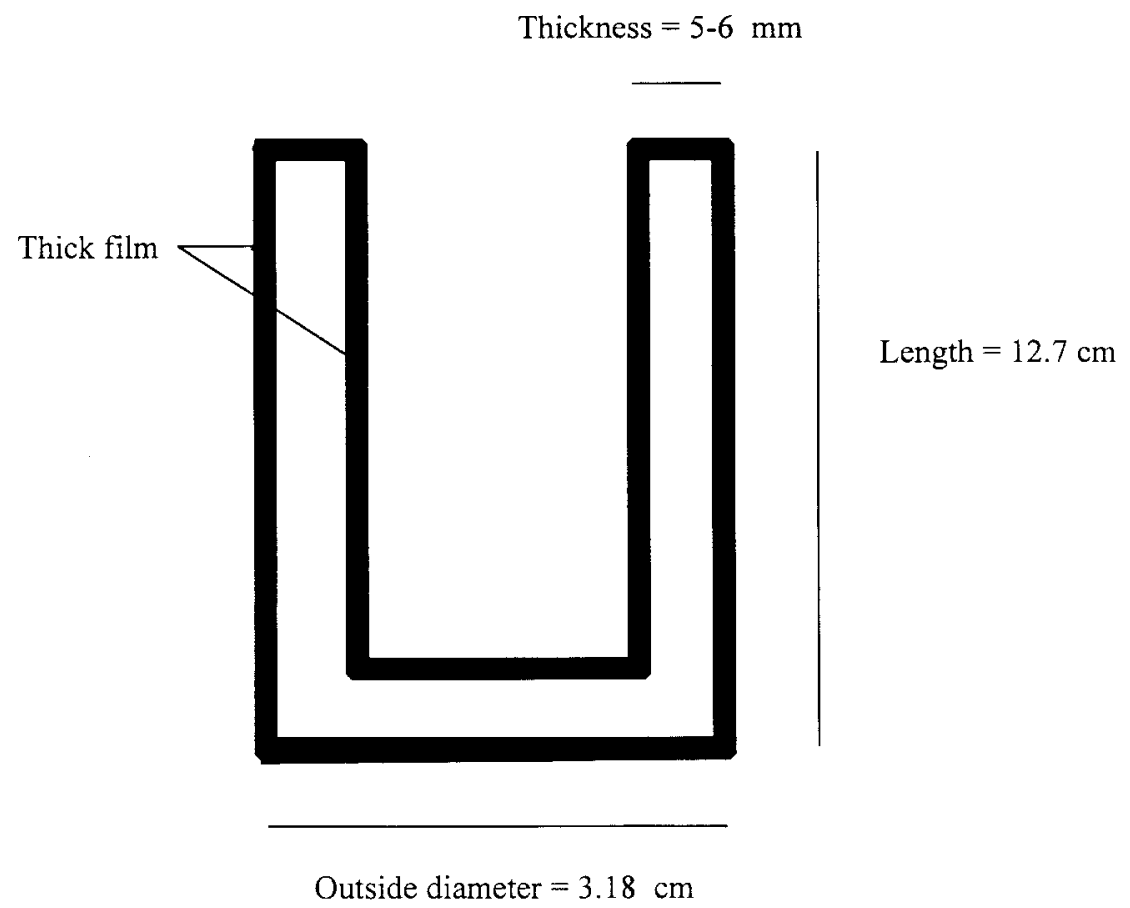

Fig. 1. Superconducting cupshield: Tl-2223 thick film on MgO.

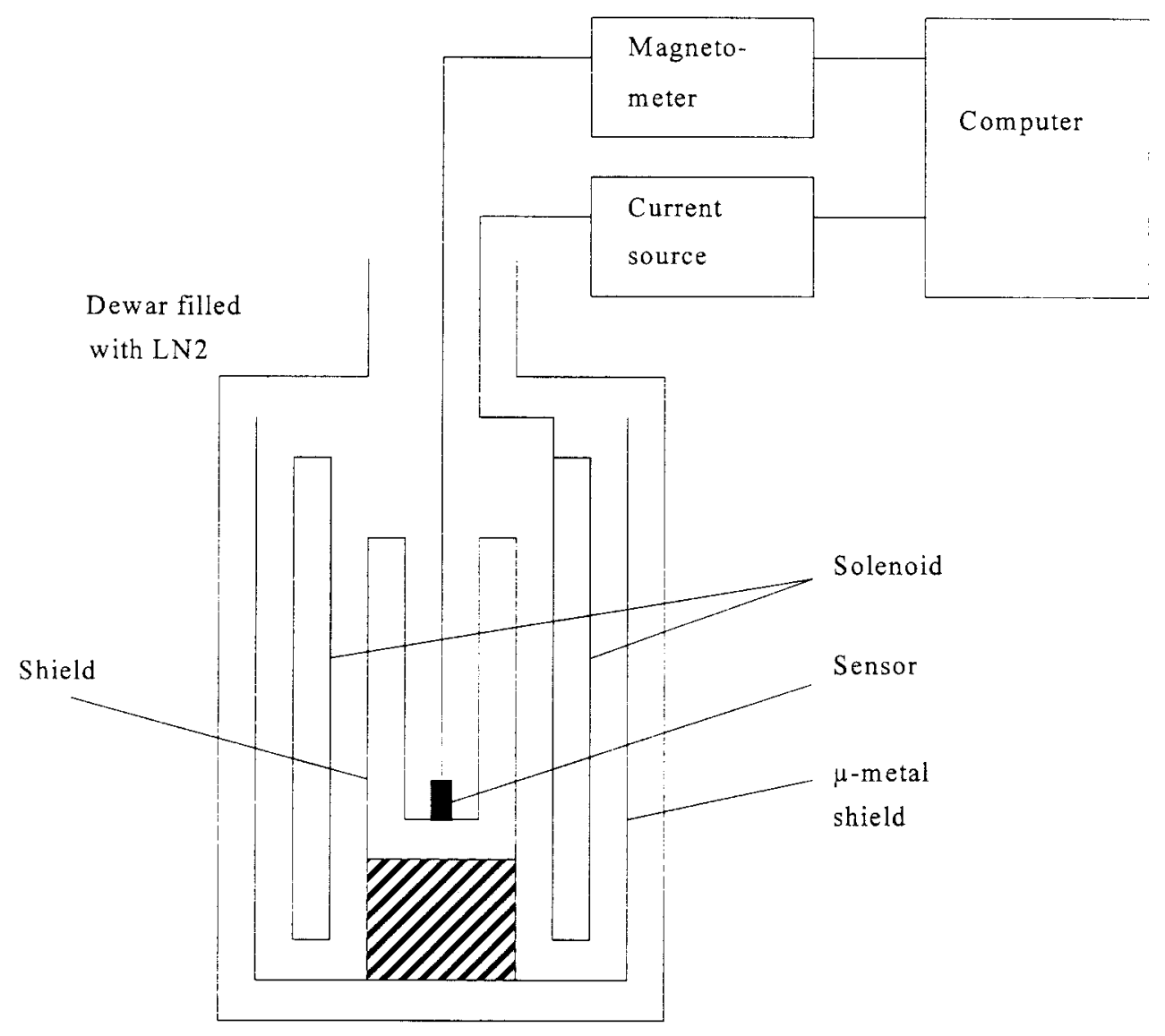

Fig. 2. Experimental setup for field measurement.

The thickness of the film $(d)$ is $\sim 1 / 2 \mathrm{~mm}$. In our analysis the shield was treated as a shield with walls of thickness $2 d$.

Our setup for measurement is shown in Fig. 2. For the field measurement, a flux gate magnetometer sensor was placed at the bottom of the shield along its axis. The magnetometer used was a Bartington MAG-01H Single Axis Fluxgate Magnetometer with circuitry as shown in Fig. 3. The shield was then placed inside a solenoid which provided an external field. 


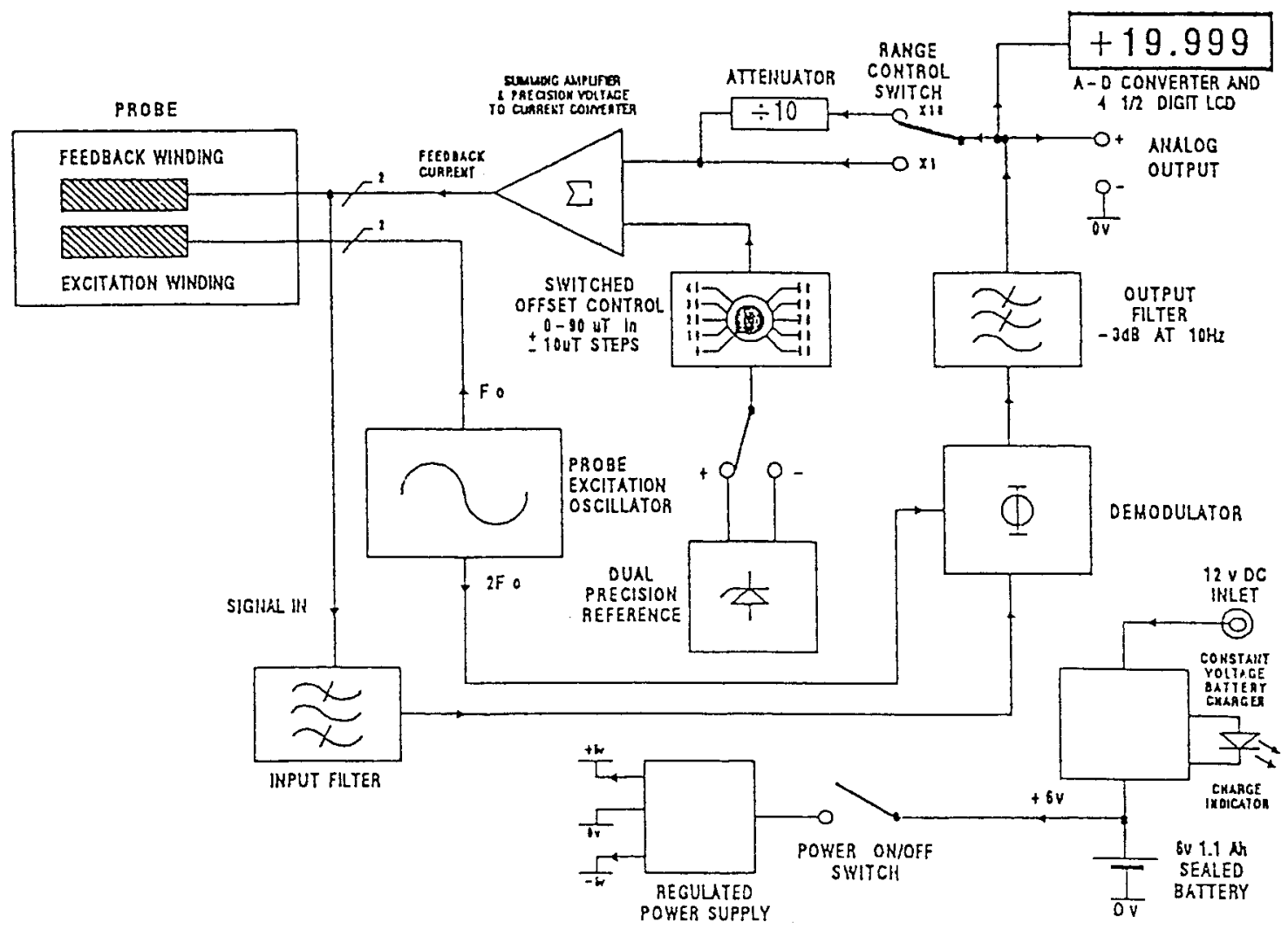

Fig. 3. Circuitry for fluxgate magnetometer (taken from [11]).

The entire arrangement was placed inside a $\mu$-metal container and immersed in liquid nitrogen inside a Dewar. It is assumed that the field in free space near the superconductor surface (the sensor location) is uniform and equal to the field on the inner surface of the cupshield.

The solenoid was connected to a current source which was controlled by a computer and calibrated to provide the appropriate axial external field $\left(H_{\text {ext }}\right)$. The sensor was connected to the magnetometer which was read by the computer at 1-s intervals.

The critical field for the shield $\left(H_{\text {crit }}\right)$ is defined as the $H_{\text {ext }}$ value for which the internal field $\left(H_{\text {int }}\right)$ becomes time dependent. We believe for a polycrystalline material $H_{\text {crit }}$ is closely associated with fluxoid movement through grain boundaries. Thus the establishment of time dependence is gradual rather than abrupt, so $H_{\text {crit }}$ actually has a range of values. For the shield used in this experiment the critical range is about $5-10 \mathrm{G}$.

The timed measurements of $H_{\text {int }}$ were taken using a computer program. Readings were taken at 1-s intervals for 700 $\mathrm{s}$ at each value of $H_{\text {ext }}$ immediately followed by $700 \mathrm{~s}$ with the direction of $H_{\text {ext }}$ reversed. $H_{\text {ext }}$ values used were 5-8 G. In an effort to reduce any initial nonzereo field present inside the shield, the first $H_{\text {int }}$ reading $\left(H_{o}\right)$ for each applied $H_{\text {ext }}$ was subtracted from all $H_{\mathrm{int}}$ readings in that cycle. $H_{\mathrm{int}}-H_{O}$ was then plotted against time.

For the high field measurements the applied fields used were $\pm 70, \pm 80$, and $\pm 90 \mathrm{G}$. The shielding device used was a bulk Tl-2223 cylindrical shell as shown in Fig. 4. Field readings were taken once every $10 \mathrm{~s}$ by the computer for a total time

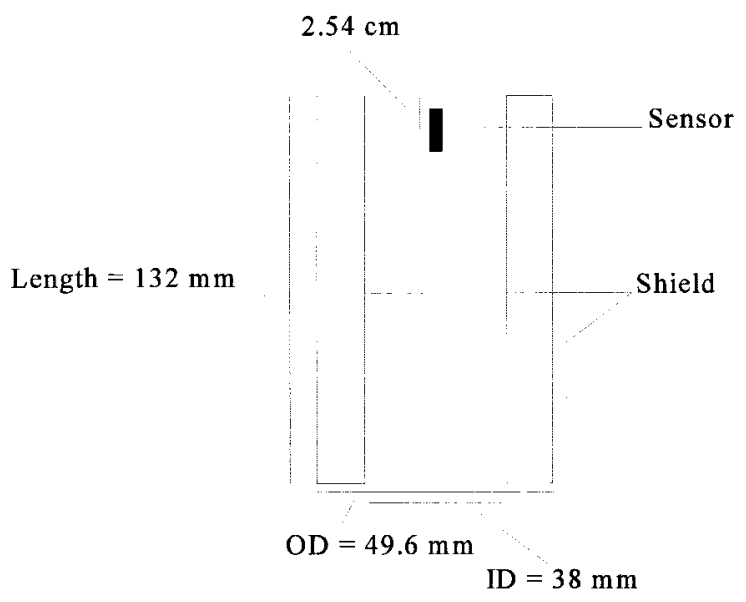

Fig. 4. Bulk Tl-2223 superconducting shield with sensor.

of $300 \mathrm{~s}$ with the sensor placed near the end of the shield as shown in Fig. 4. The same field source-Dewar-sensor setup was used as for the cupshield. Parameters associated with the shield are given as follows:

$$
\begin{aligned}
& \text { Length }=132 \mathrm{~mm} \\
& \text { Inside diameter }=38 \mathrm{~mm} \\
& \text { Outside diameter }=49.6 \mathrm{~mm} \\
& \text { Mass }=488.9 \mathrm{~g} \\
& \text { Density }=4.64 \mathrm{~g} / \mathrm{cm}^{3} .
\end{aligned}
$$




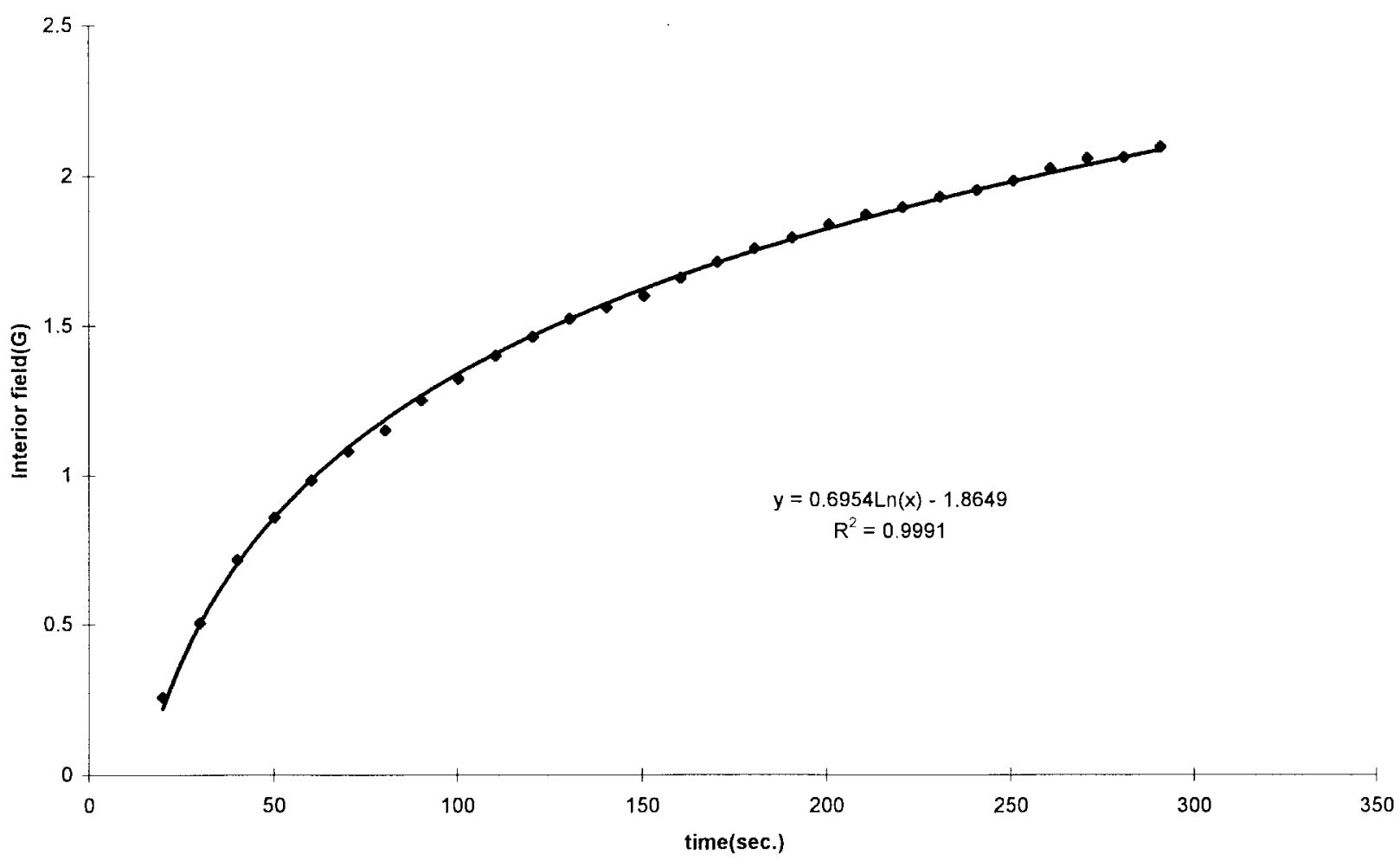

Fig. 5. Interior field versus time for bulk shield at $80 \mathrm{G}$.

The interior field has a logarithmic time dependence as shown in Fig. 5.

\section{EXPERIMENTAL RESULTS}

Empirical curves of the form $H_{\mathrm{int}}-H_{0}=\sum_{n} A_{n} e^{-t / \tau_{n}}+$ $H_{f}$ were fitted by trial and error to plots of experimental cupshield data. The equations for the empirical curves are given and plotted with data in Fig. 6. Fig. 7 shows a plot of the difference between the experimental and empirical curves. The largest differences tend to occur at low time values. We believe this is probably due to error introduced in synchronization between the external source and the internal field reading.

It is interesting to compare the empirical results with solutions of the diffusion equation. Diffusion through the cylindrical cupshield must be subject to the following conditions:

$$
\begin{aligned}
& H(r=0) \text { is finite } \\
& H(t \rightarrow \infty)=H_{f}=\text { constant } \\
& H\left(r=R_{\text {out }}\right)=H_{f}=\text { constant } \\
& R_{\text {out }}=\text { outer radius } \\
& \text { Cylindrical symmetry. }
\end{aligned}
$$

Any solution of the diffusion equation that satisfies these conditions has the following form:

$$
H(r, t)=\sum_{n} A_{n} J_{o}\left(\alpha_{n} r / R_{\mathrm{out}}\right) e^{-t / \tau_{n}}+H_{f}
$$

The measurements taken in this experiment were taken at $r=0$ so it is appropriate to look at $H(0, t)$

$$
H(0, t)=\sum_{n} A_{n} e^{-t / \tau_{n}}+H_{f}
$$

Recall that the empirical curves, $H_{\text {int }}-H_{0}=$ $\sum_{n} A_{n} e^{-t / \tau_{n}}+H_{f}$, have the same form as $H(0, t)$ in (3). In order to satisfy the diffusion equation, however, the empirically determined $\tau_{n}$ 's must also satisfy the restriction given by the diffusion equation

$$
\begin{aligned}
& \text { Diffusion equation: } \frac{\partial H}{\partial t}=c^{2} \nabla^{2} H \\
& \text { Replacing } \nabla^{2} H=\frac{1}{r} \frac{\partial H}{\partial r}+\frac{\partial^{2} H}{\partial r^{2}}
\end{aligned}
$$
assuming $H$ is independent of $\phi$ and $z$ in cylindrical coordinates.

We obtain

$$
c^{2} \nabla^{2} H=\sum_{n}-\left(c \alpha_{n} / R_{\text {out }}\right)^{2} A_{n} J_{o}\left(\alpha_{n} r / R_{\text {out }}\right) e^{-t / \tau_{n}}
$$

The left side of (4) is given by

$$
\frac{\partial H}{\partial t}=\sum_{n}-\left(1 / \tau_{n}\right)^{2} A_{n} J_{o}\left(\alpha_{n} r / R_{\mathrm{out}}\right) e^{-t / \tau_{n}} .
$$

Thus equating the expressions in (6) and (7), we must have

$$
\frac{\alpha_{n}}{\sqrt{\tau_{n}}}=\frac{R_{\mathrm{out}}}{c}=\text { constant. }
$$

Therefore, for a given $H_{\text {ext }}$, there should be an $\alpha_{n}$ for every $\tau_{n}$ such that $\alpha_{n} \sqrt{\tau_{n}}=$ constant. The empirical $\tau_{n}$ 's obey 


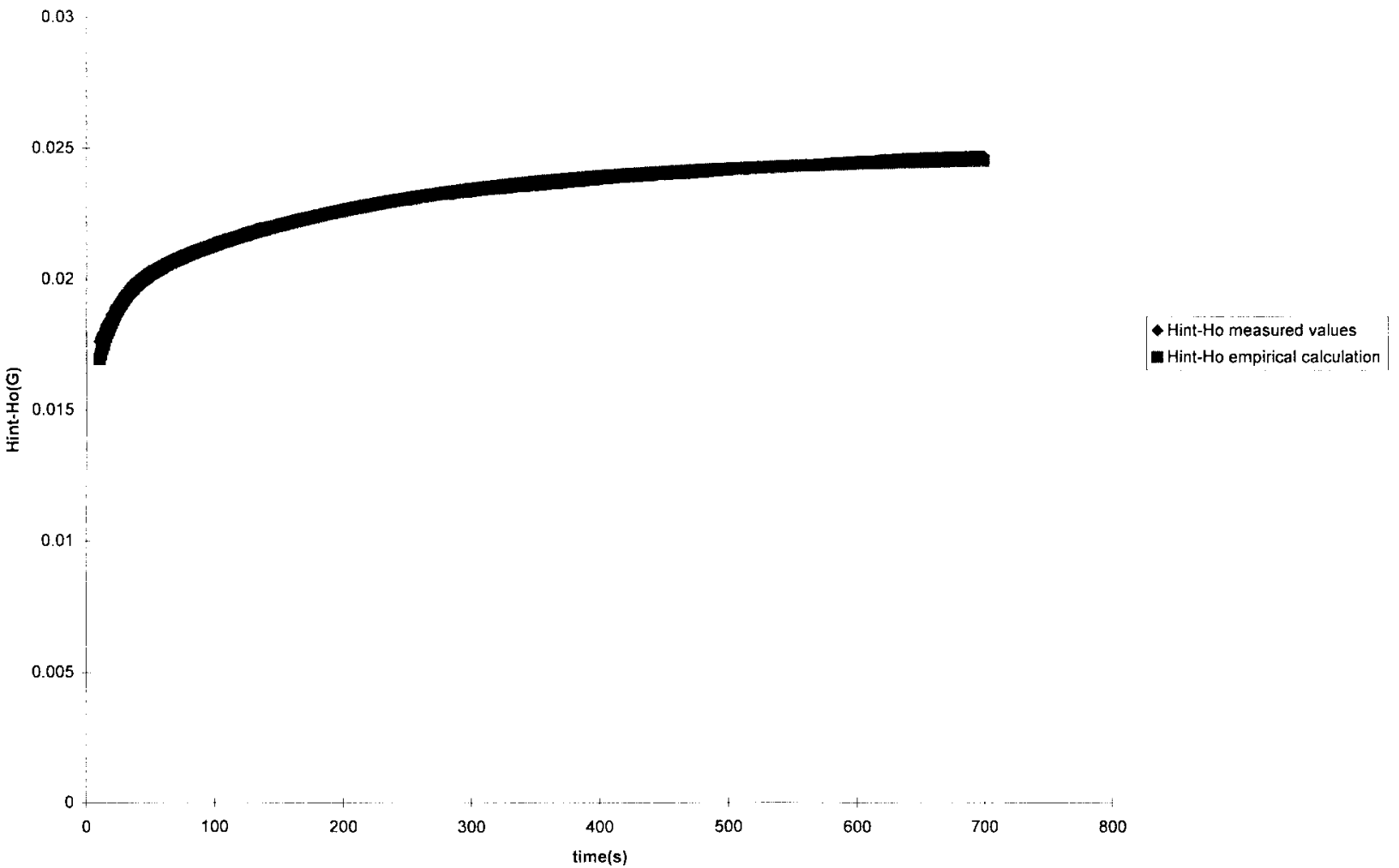

Fig. 6. Plot of empirical curve (from Table I) and measured values of $H_{\mathrm{int}}-H_{O}(\mathrm{G})$ versus time (s) at 5 G.

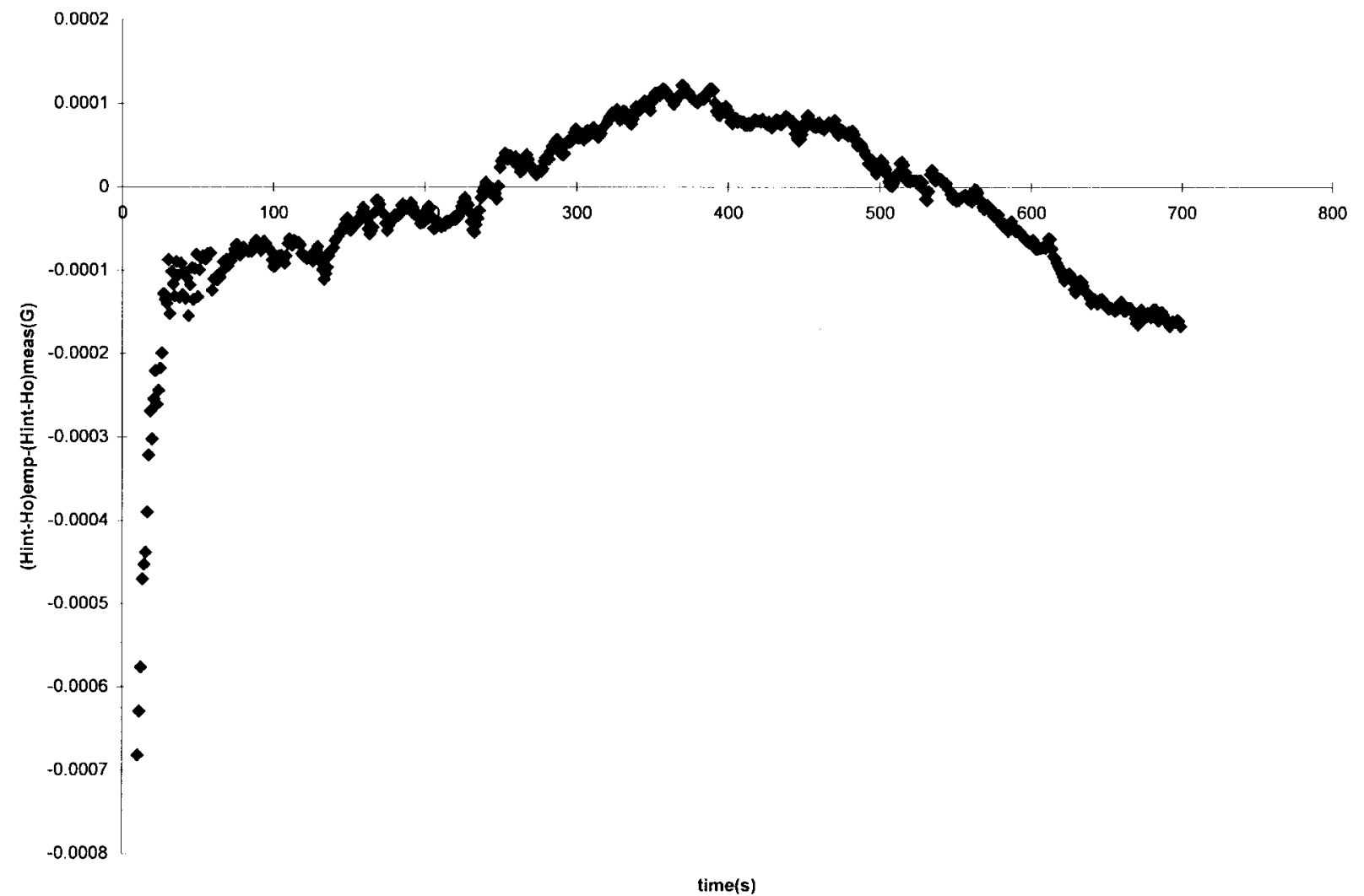

Fig. 7. Difference between empirical curve and measured values for $H_{\mathrm{int}}-H_{o}(\mathrm{G})$ versus time (s) at $5 \mathrm{G}$. 
TABLE I

\begin{tabular}{|c|c|c|c|}
\hline \multicolumn{4}{|l|}{ For $\mathrm{H}_{\mathrm{ext}}=5 \mathrm{G}$ : } \\
\hline \multicolumn{4}{|c|}{$\mathrm{H}(0, \mathrm{t})=\left(-0.0246 \mathrm{e}^{-3 / 2}\right) \mathrm{e}^{-\mathrm{v} 200}-0.005 \mathrm{e}^{-\mathrm{v} / 15}+0.0246(\mathrm{t}$ in $\mathrm{s}, \mathrm{H}$ in $\mathrm{G})$} \\
\hline$\tau_{n}(s)$ & $\mathrm{n}$ & $\alpha_{n}$ & $\alpha_{n} \sqrt{\tau_{n}}\left(\mathrm{~s}^{1 / 2}\right)$ \\
\hline 200 & 1 & 2.40483 & 34.01 \\
\hline 15 & 3 & 8.65373 & 33.52 \\
\hline \multicolumn{4}{|l|}{ For $\mathrm{H}_{\mathrm{ext}}=6 \mathrm{G}$ : } \\
\hline \multicolumn{4}{|c|}{$H(0, t)=\left(-0.0856 \mathrm{e}^{-300 / 201}\right) \mathrm{e}^{-\mathrm{v} 201}-0.017 \mathrm{e}^{-\mathrm{v} 15}+0.0856(\mathrm{t}$ in $\mathrm{s}, \mathrm{H}$ in $\mathrm{G})$} \\
\hline$\tau_{n}(s)$ & $\mathrm{n}$ & $\alpha_{n}$ & $\alpha_{n} \sqrt{\tau_{n}\left(s^{1 / 2}\right)}$ \\
\hline 201 & 1 & 2.40483 & 34.09 \\
\hline 15 & 3 & 8.65373 & 33.52 \\
\hline
\end{tabular}

For $\mathrm{H}_{\mathrm{exl}}=7 \mathrm{G}$ :

\begin{tabular}{|c|c|c|c|}
\hline \multicolumn{4}{|c|}{$\begin{aligned} \mathrm{H}(0, \mathrm{t})=\left(-0.364 \mathrm{e}^{-44 / 26}\right) \mathrm{e}^{-\mathrm{v} 260}-0.08 \mathrm{e}^{-\mathrm{v} 25}+0.01 \mathrm{e}^{-t 20}+(0.01 \mathrm{e}) \mathrm{e}^{-v 10} \\
+\left(0.006 \mathrm{e}^{10 / 15}\right) \mathrm{e}^{-v 15}-0.006 \mathrm{e}^{-\mathrm{v} 600}+0.369(\mathrm{t} \text { in } \mathrm{s}, \mathrm{H} \text { in } \mathrm{G})\end{aligned}$} \\
\hline$\tau_{n}(s)$ & $\mathrm{n}$ & $\alpha_{n}$ & $\alpha_{n} \sqrt{\tau_{n}\left(s^{1 / 2}\right)}$ \\
\hline 600 & 2 & 5.52008 & 135.2 \\
\hline 260 & 3 & 8.65373 & 139.5 \\
\hline 25 & 9 & 27.49348 & 135.7 \\
\hline 20 & 10 & 30.63461 & 137.0 \\
\hline 15 & 11 & 33.77582 & 130.8 \\
\hline 10 & 14 & 43.19979 & 136.6 \\
\hline
\end{tabular}

For $\mathrm{H}_{\mathrm{ext}}=8 \mathrm{G}$ :

$$
\begin{array}{cccc}
H(0, t)=\left(-1.01 \mathrm{e}^{-310 / 178}\right) \mathrm{e}^{-v 178}+1.01(\mathrm{t} \text { in } \mathrm{s}, \mathrm{H} \text { in } \mathrm{G}) \\
\\
\begin{array}{cccc}
\tau_{\mathrm{n}}(\mathrm{s}) & \mathrm{n} & \alpha_{\mathrm{n}} & \alpha_{\mathrm{n}} \sqrt{\tau_{\mathrm{n}}\left(\mathrm{s}^{1 / 2}\right)} \\
178 & 2 & 5.52008 & 73.65
\end{array}
\end{array}
$$

this restriction well as shown in Table $\mathrm{I}$, indicating that the diffusion equation is well satisfied for $H_{\text {ext }}<10 \mathrm{G}$. Values for $\alpha_{n}$ were taken from Abramowitz and Stegun [12].

For $H_{\text {ext }}>60 \mathrm{G}$ bulk shield data, empirical curves of the form $H_{\text {int }}=H_{a} \ln t+H_{b}$ were fitted to experimental plots of applied field versus time. The equations for these empirical curves are given and plotted with the data in Fig. 5.

At this point we will show that such a logarithmic time dependence at the higher applied field is still compatible with the diffusion model, since the time dependence near the critical threshold appears to have the form of a summation of exponentials. At a higher applied field, more terms must be included, hence the summation becomes an integral

$$
H(0, t)-H_{f}=\int_{0}^{\infty} P(\sigma) e^{-t \sigma} d \sigma, \quad \text { where } \sigma \sim 1 / \tau
$$

Since $\tau_{n}$ is given by the roots of $J_{o}$, it is inversely proportional to $\epsilon_{n}$, the magnetic energy eigenvalue. This can also be written as an integral over an energy distribution, $D(\epsilon)$

$$
H(0, t)-H_{f}=\int_{\epsilon_{0}}^{\infty} D(\epsilon) e^{-\epsilon t / \hbar} d \epsilon
$$

where $\epsilon_{0}$ is the minimum fluxoid energy. It is physically reasonable to assume that the interior field is a superposition of electromagnetic waves with a Planck energy distribution

$$
D(\epsilon)=\frac{e^{-\beta \epsilon}}{1-e^{-\beta \epsilon}} \approx \frac{e^{-\beta \epsilon}}{\beta \epsilon} \quad(\beta \epsilon \text { small }) .
$$

Equation (10) then becomes

$$
\begin{aligned}
H(0, t)-H_{f}= & \int_{\epsilon_{0}}^{\infty} \frac{e^{-\beta \epsilon}}{\beta \epsilon} e^{-\epsilon t / \hbar} d \epsilon \\
= & \frac{1}{\beta} \int_{\epsilon_{0}}^{\infty} \frac{e^{-(\beta \epsilon+\epsilon t / \hbar)}-e^{-\beta \epsilon}}{\epsilon} d \epsilon \\
& +\int_{\epsilon_{0}}^{\infty} \frac{e^{-\beta \epsilon}}{\beta \epsilon} d \epsilon
\end{aligned}
$$

We then assume that $\epsilon_{0}$ is near zero (but not equal to zero). To consider the effect of this assumption, we consider the result if we perform the integrations in (12), changing the limits to zero and $\epsilon_{0}$. The first integral gives a very small result, and the second integral gives a large negative result. Equation (12) can therefore be modified to

$$
\begin{aligned}
H(0, t)-H_{f} \approx & \frac{1}{\beta} \int_{0}^{\infty} \frac{e^{-(\beta \epsilon+\epsilon t / \hbar)}-e^{-\beta \epsilon}}{\epsilon} d \epsilon \\
& +\int_{\epsilon_{0}}^{\infty} \frac{e^{-\beta \epsilon}}{\beta \epsilon} d \epsilon .
\end{aligned}
$$

Performing the first integration and noting that the second integral is a constant yields

$$
H(0, t)=-(1 / \beta) \ln [1+t / \beta \hbar]+\text { constant }
$$

which shows a logarithmic time-dependence at higher applied fields.

\section{SUMMARY AND INTERPRETATION}

The time dependence of the internal field of a high $T_{c}$ shielding device in an axial applied field is modeled as being diffusive. The diffusion model proposed involves flux lines between the superconducting grains in the field regime of the Meissner effect. Flux motion is statistical but tends to be in the direction of decreasing flux density, much like the motion of chemical species diffusing in the direction of decreasing concentration. Measurements taken using a thick film Tl-2223 shield near the critical threshold support such a model. A logarithmic time dependence seen in bulk Tl-2223 shields at higher applied fields is shown to be theoretically compatible with flux diffusion.

While Kim-Anderson flux creep has a logarithmic time dependence, it also requires that the flux density be large enough and the temperature be low enough to form a lattice (and thus bundles). This can happen only if the applied field is greater than $H_{C 1}$ (our applied field is much lower than this) and the temperature is very low (much lower that $77 \mathrm{~K}$ which is the temperature range of our experiment). Our logarithmic time dependence can be experimentally distinguished from that of Kim-Anderson flux creep and that of avalanche behavior because these two mechanisms occur on a much shorter time scale $\left(\sim 10^{-11}\right.$ to $\left.10^{-5} \mathrm{~s}\right)$ [4] than we observe in our experiment $\left(\sim 10^{2} \mathrm{~s}\right)$. Because of the above field, temperature, and time scale criteria, we conclude that it is unlikely that 
the flux lines in our regime of field and temperature move according to Kim-Anderson flux creep or avalanche flux motion. We have proposed a model of flux diffusion between grains that could theoretically occur in our regime of field and temperature, and that is consistent with our data (our data satisfy the diffusion equation).

\section{ACKNOWLEDGMENT}

The authors would like to thank N. S. Alzayed, W. N. Kang, and D. F. Lu for their assistance in computer programming and Z. Ying for her assistance in sample preparation.

\section{REFERENCES}

[1] Y. B. Kim and M. J. Stephen, "Flux flow and irreversible effects," in Superconductivity, vol. 2, R. D. Parks, Ed. New York: Marcel Dekker, 1969, pp. $1107-1165$.

[2] P. W. Anderson, "Theory of flux creep in hard superconductors," Phys. Rev. Lett., vol. 9, pp. 309-311, Oct. 1962.

[3] Y. B. Kim, C. F. Hempstead, and A. R. Strnad, "Critical persistent currents in hard superconductors," Phys. Rev. Lett., vol. 9, pp. 306-309, Oct. 1962.

[4] M. Tinkham, Introduction to Superconductivity. International Series in Pure and Applied Physics. New York: McGraw-Hill, 1996.

[5] S. Field, J. Witt, F. Nori, and X. Ling, "Superconducting vortex avalanches," Phys. Rev. Lett., vol. 74, pp. 1206-1209, Feb. 1995

[6] P. G. de Gennes, Superconductivity of Metals and Alloys. New York: W. A. Benjamin, 1966.

[7] A. M. Campbell and J. E. Evetts, "Flux vortices and transport currents in type II superconductors," Adv. Phys., vol. 21, pp. 199-428, Mar. 1972.

[8] D. Shi and Z. Wang, "Avalanche flux dynamics in the vortex state of type-II superconductors," IEEE Trans. Appl. Superconduct., vol. 5, pp. 1721-1724, June 1995.

[9] M. Yang, Y. H. Kao, Y. Xin, and K. W. Wong, "Chemical doping and intergranular magnetic-field effects in bulk thallium-based superconductors," Phys. Rev. B, vol. 50, pp. 13,653-13,658, Nov. 1994.

[10] K.-H. Muller, "AC susceptibility of high temperature superconductors in a critical state model," Physica C, vol. 159, pp. 717-726, 1989.

[11] Bartington Instruments, Ltd., Operation Manual for MAG-01 and MAG01H Single Axis Fluxgate Magnetometers. Bartington, 1992.

[12] M. Abramowitz and I. A. Stegun, Eds., Handbook of Mathematical Functions with Formulas, Graphs, and Mathematical Tables, Applied Mathematics Series 55, National Bureau of Standards, U.S. Department of Commerce, 1972

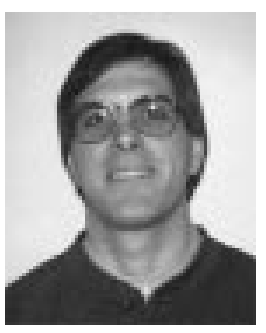

A. D. Havenhill received the B.S. degree in geophysics from the University of Kansas, Lawrence, in 1988, the M.S. degree in geology from the University of Georgia, Athens, in 1992, and the Ph.D. degree in physics from the University of Kansas in 1997.

$\mathrm{He}$ is currently a member of the faculty of the University of South Dakota. His research interests include high temperature superconductivity and the behavior of HTS materials at applied magnetic fields.

Dr. Havenhill is a member of Sigma Pi Sigma, Alpha Chi Sigma, and Phi Kappa Phi.

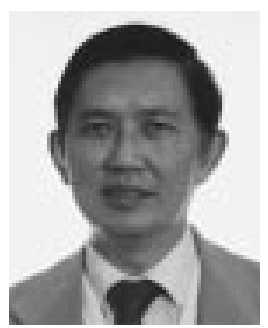

K. W. Wong received the B.S. degree from Duke University, Durham, NC, in 1959 and the M.S and Ph.D. degrees from Northwestern University, Chicago, IL, in 1960 and 1963, respectively.

He was an Assistant Professor, then Associate Professor, at the University of Kansas, Lawrence, from 1964 to 1968 and 1968 to 1972, respectively. He became a Professor in 1972. He has been Director of the University of Kansas High Tc Laboratory since 1990 and is also a Founder of Midwest Superconductivity, Inc., Lawrence, KS.

Dr. Wong is a Member of the American Physical Society, AAAS, the New York Academy of Science, and the Hong Kong Academy of Science.

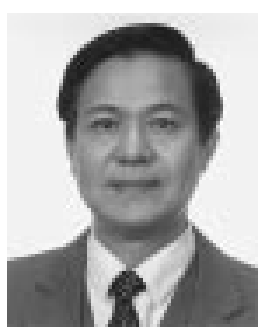

C. X. Fan was an Associate Professor of Applied Physics and Electrical Engineering at Fudan University, China. He is currently a Senior Scientist of Midwest Superconductivity, Inc., Lawrence, KS. His research interests include applications of superconductivity, especially SQUID, microwave detectors, and other sensors. 